\title{
Numerical analysis of mixed convection in air in an inclined channel with a moving plate
}

\author{
A. Andreozzi, N. Bianco, G. Lacasa \& V. Naso \\ DETEC, Università di Napoli FEDERICO II, Napoli, Italy
}

\begin{abstract}
A numerical investigation of mixed convection in air due to the interaction between a buoyancy flow and a moving plate induced flow in an inclined channel is presented. The moving plate has a constant velocity and is unheated whereas the other channel wall is stationary and heated at uniform heat flux. The numerical analysis is obtained by means of the commercial Fluent code. The effects of the inclination angle, channel spacing, heat flux and moving plate velocity are investigated and results in terms of the heated channel wall and moving plate temperatures are given.

Keywords: mixed convection, numerical analysis, inclined channel, moving plate.
\end{abstract}

\section{Introduction}

Mixed convection due to moving surfaces is very important in a wide variety of materials processing systems, such as soldering, welding, extrusion of plastics and other polymeric materials, hot rolling, cooling and/or drying of paper and textiles, Chemical Vapor Deposition (CVD), composite materials manufacturing, as reviewed in [1-4] .

Mixed convection as a result of buoyancy and motion of one of the channel walls has received little research attention and few guidelines are available for choosing the best performing channel configuration. A study on this topic is presented in [5], where the conjugate mixed convection and conduction heat transfer due to a continuous moving plate in a parallel channel flow was numerically investigated. Results showed that the effect of thermal buoyancy was stronger when the plate moved vertically than when it moved horizontally. The plate velocity affected the overall heat transfer significantly. 
A numerical investigation of mixed convection in air due to the interaction between a buoyancy flow and a moving plate induced flow in a vertical channel was carried out in [6]. The moving plate had a constant velocity and moved along the buoyancy force direction whereas the principal walls of the channel were heated at uniform heat flux. Results showed that the moving plate effects were more significant when the channel gap was lower, for lower moving plate velocity values. For higher moving plate velocity values these effects were more considerable for all channel gap values and for lower heat flux values. In [7] the authors studied the same configuration with the moving plate moved in the opposite direction with respect to the buoyancy force. The wall temperature profiles allowed to observe different behaviors of the flow motion inside the channel, a buoyancy flow, a forced flow and a transition flow related to the velocity of moving plate. An experimental analysis of mixed convection in air in a vertical channel was presented in [8]. One of the channel walls was unheated and moved at a constant velocity, whereas the other one was heated at a uniform heat flux. Two configurations were investigated: Assisting, where the effect of the moving wall superposed to natural convection and Opposing, where the effect of the moving wall opposed natural convection. A numerical investigation of mixed convection in air in horizontal parallel walled channels with moving lower plate was carried out in [9]. The moving lower plate had a constant velocity and it was adiabatic, whereas the upper one was heated at uniform heat flux. For stationary condition of lower plate, a typical $C$-loop inside the horizontal channel was detected. Different flow motions were observed in the analyzed configuration, depending on the heat flux values and the distance between the heated upper stationary plate and the lower adiabatic moving plate.

It seems that there is a lack of information in the open literature on mixed convection in parallel plate channels with a continuous moving plate in spite of its importance in heat treatment processes.

In the present study a numerical investigation of mixed convection in air due to the interaction between the buoyancy flow and the forced flow induced by a moving plate in an inclined channel is carried out. The moving plate is unheated and it moves at a constant velocity whereas the other wall of the channel is stationary and heated at a uniform heat flux. The effect of radiative heat transfer is neglected. The effects of the inclination angle, channel spacing, heat flux and moving plate velocity are investigated and results in terms of the heated channel wall and moving plate temperatures are given.

\section{Mathematical description and numerical procedure}

The investigated system consists of an inclined channel made up of a moving plate and a stationary plate. The moving plate, in the following also called "belt", moves at a constant velocity, it is unheated and it is assumed to be zero thickness. The stationary channel wall is conductive and heated at a uniform heat flux, $q_{w}$, and its thickness is $s$. The length of the channel is $L_{p}$ and its spacing is $b$. The working fluid is air. Mixed convective flow in the inclined channel is assumed 


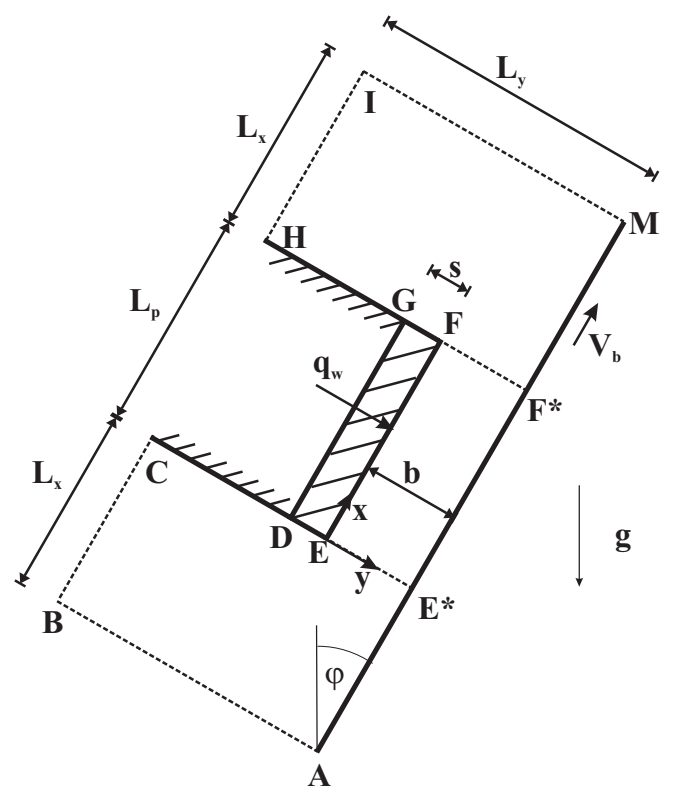

Figure 1: Computational domain.

to be incompressible. Moreover the system is assumed to be wide enough along the third coordinate to allow a 2D approximation. All thermophysical properties of the fluid are assumed to be constant, except for the dependence of density on the temperature (Boussinesq approximation). The thermophysical properties of the fluid are evaluated at the ambient temperature, $T_{0}$, which is assumed to be $300 \mathrm{~K}$ in all cases.

The governing equations for the fluid region in steady state and turbulent regime are time-averaged mass, Navier-Stokes and energy equations. A two-dimensional conduction model in the heated wall is employed whereas the transport equations for $k$ and $\varepsilon$ are formulated using the RNG $k-\varepsilon$ model.

An enlarged computational domain has been chosen and it is shown in Fig. 1. It is made up of the inclined channel and of two reservoirs of dimensions $L_{x}$ and $L_{y}$, downstream and upstream of it, that simulate the free-stream conditions of the flow far away from the region thermally disturbed by the heated channel wall. The moving plate extends from the lower to the upper reservoir and its height is $L_{b}=L_{p}+2 L_{x}$. The imposed boundary conditions are:

- uniform heat flux and no-slip condition on the stationary channel wall;

- adiabatic wall and no-slip condition on the other solid walls;

- pressure inlet or pressure outlet conditions on the open-boundaries;

- continuity of the heat flux on the wall EF, FG and ED.

The commercial Fluent CFD code was employed to solve the governing equations. The SIMPLE scheme was chosen to couple pressure and velocity. The 
converging criteria were $10^{-5}$ for the residuals of the velocity components, $10^{-8}$ for the residuals of the energy and $10^{-6}$ for the residuals of $k$ and $\varepsilon$.

Preliminary results showed that the chosen turbulent model was suitable also for laminar flow. Several preliminary tests were carried out to take into appropriate account the effects of turbulence parameters, $k$ and $\varepsilon$, at the inlet. Assuming that the fluid comes from a quiescent zone, the distributions of $k$ and $\varepsilon$ were taken as uniform and equal to $10^{-6}$. Moreover, the static pressure upstream and downstream of the channel is set equal to the ambient pressure far away from the region of the thermal disturbance induced by the heated channel wall [10].

In order to analyze the grid independence of the numerical solution, different grid sizes were employed. The domain discretization was performed in such a way as to have a larger number of nodes in the channel region where the highest gradients were expected. A uniform grid was employed along the $A E^{*}, E^{*} F^{*}$, $E E^{*}$ and $D E$ sides, whereas an exponential law was used along $C D$. In this case the subinterval length decreased toward the channel. Preliminary tests were carried out to evaluate the dependence of the solution on the employed grid.

An analogous analysis has been carried out to set the optimal reservoirs dimensions; $L_{x}=0.400 m$ and $L_{y}=4 b+s$ have been chosen.

\section{Results and discussion}

In the following results are presented for air, for a heated plate length $L_{p}=$ $0.406 \mathrm{~m}$, for channel spacings $b=10$ and $20 \mathrm{~mm}$, for wall heat fluxes $q_{w}=30$ and $120 \mathrm{~W} / \mathrm{m}^{2}$, for inclination angles in the range $0^{\circ}-90^{\circ}$ and for belt velocities $V_{b}= \pm 0.1$ and $\pm 1 \mathrm{~m} / \mathrm{s}$. The thickness of the heated wall is $3.2 \mathrm{~mm}$.

Temperature profiles of the heated wall and the unheated moving plate, referred to the ambient temperature, as a function of the coordinate along the channel, at different inclination angles, channel spacings, wall heat fluxes, belt velocities are presented in the following. Two configurations have been investigated: Assisting, where the vertical component of the belt velocity is opposed to the acceleration due to the gravity, and Opposing, where the afore mentioned component has the same direction as that of the acceleration due to the gravity. In the Opposing configuration two different flow patterns can occur, according to the ratio between the buoyancy force from the heated wall and the drag force from the moving unheated plate. When the former prevails upon the latter, the air in the channel flows upward in the main, in the other case air flows downward in the main. Even though in many cases air flows neither upward nor downward everywhere in the channel, one can notice that in the Opposing configuration there is a critical value of the belt velocity, named transition velocity, at which some stagnation of the air flow occurs in the channel and, as a result, maximum wall temperatures are attained.

Heated wall temperature profiles, referred to an ambient temperature equal to $300 K$, for different values of the channel spacing, the wall heat flux, the velocity of the unheated moving plate and the inclination angle of the channel, both for the Assisting and the Opposing configurations, are presented in Figs. 2-4. 

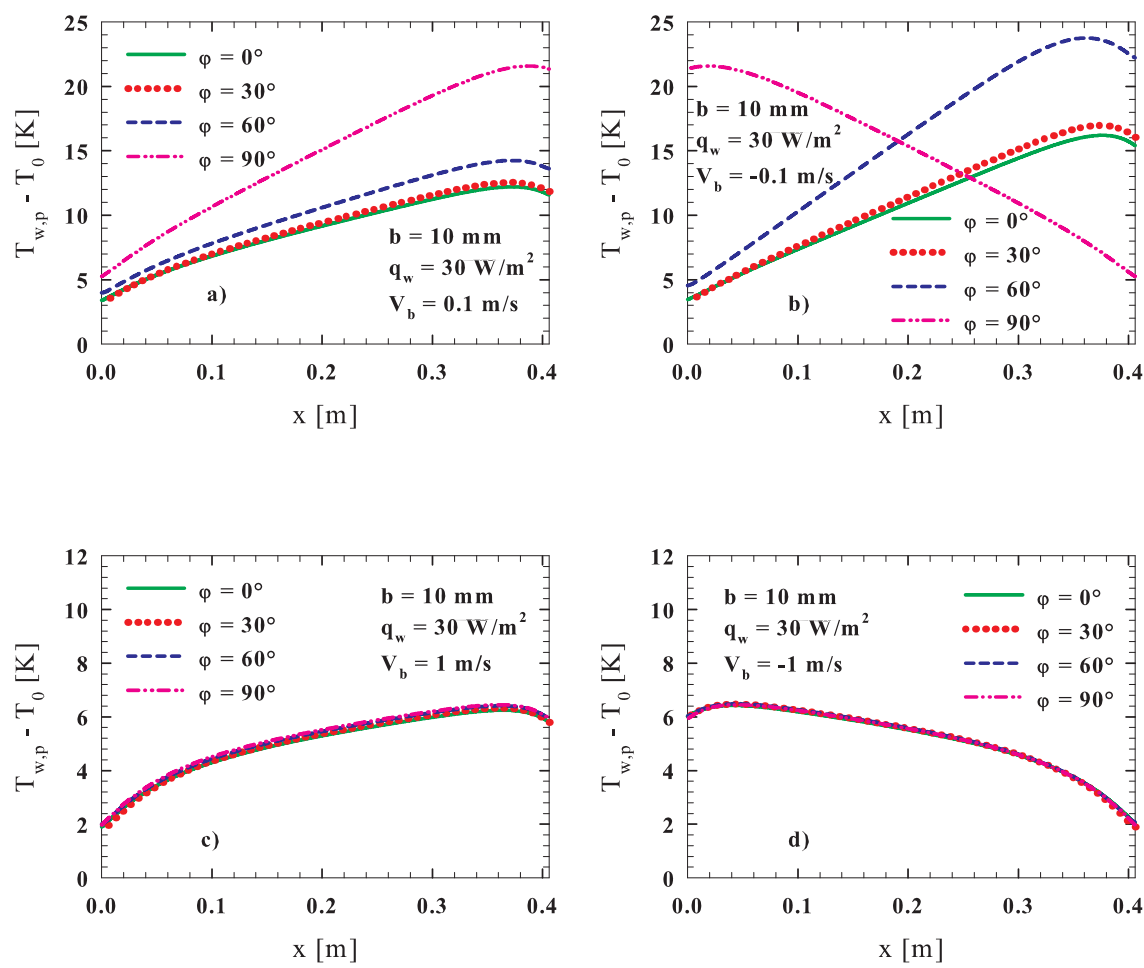

Figure 2: Heated wall temperature profiles vs axial coordinate for different inclination angles, $b=10 \mathrm{~mm}$ and $q_{w}=30 \mathrm{~W} / \mathrm{m}^{2}$ : (a) $V_{b}=0.1 \mathrm{~m} / \mathrm{s}$; (b) $V_{b}=-0.1 \mathrm{~m} / \mathrm{s}$; (c) $V_{b}=1 \mathrm{~m} / \mathrm{s}$; (d) $V_{b}=-1 \mathrm{~m} / \mathrm{s}$.

Figure 2(a), for a $10 \mathrm{~mm}$ channel gap, a $30 \mathrm{~W} / \mathrm{m}^{2}$ wall heat flux, a $0.1 \mathrm{~m} / \mathrm{s}$ belt velocity in the Assisting mode, shows that the larger the axial coordinate the larger the wall temperature, whichever the inclination angle. As a consequence, an ascending air flow occurs in all inclined channels whereas air flows from the left end section of the horizontal channel. Figure 2(a) points out that the larger the inclination angle the larger the wall temperature, since the larger the angle the smaller the contribution of the buoyancy force to the ascending air flow and, therefore, the higher the wall temperature. We can notice that differences in wall temperatures profiles at $\varphi=0^{\circ}$ and $\varphi=30^{\circ}$ (nearly $1 K$ ) are smaller than those at $\varphi=60^{\circ}$ and $\varphi=90^{\circ}$ (up to $8 \mathrm{~K}$ ), the maximum temperature of the heated wall above the ambient temperature being nearly $20 \mathrm{~K}$.

Figure 2(b) refers to the same parameters as those in the previous figure apart from the Opposing configuration and shows that in inclined channels the velocity of the air is smaller than the transition velocity, since the continuous increase in wall temperatures from the inlet to the exit section of the channels indicates a 
prevailing upward flow of the air. It is plain that, in the horizontal channel, wall temperature profiles both in the Assisting and Opposing mode are symmetric as a result of the geometric and thermal symmetry in a horizontal channel. Like in the Assisting mode also in the Opposing one the larger the tilting angle the larger the wall temperature, because of the decrease in the component of the buoyancy force along the channel axis. Observing Figs. 2(a) and 2(b) allows to remark that wall temperatures are larger in the Opposing mode than in the Assisting one, apart at $\varphi=90^{\circ}$, since the descending belt motion slows up the ascending air flow. The dependence of wall temperatures on the inclination angle in the Opposing mode is more marked than in the Assisting mode. As a matter of fact, larger wall temperatures increase the role of buoyancy flow versus forced flow and, therefore, the effect of the tilting angle.

In the Opposing configuration at any combinations of investigated parameters there is an inclination angle at which buoyancy forces equal the forced flow and the belt moves at the transition velocity. In the investigated case the afore mentioned tilting angle falls in the $\varphi=60^{\circ}-90^{\circ}$ range. Below the inclination angle at which the belt velocity equals the transition velocity the larger the angle the larger the wall temperature whereas for larger tilting angles increasing the angle decreases wall temperatures.

Predictions for a larger belt velocity are presented in Figs. 2(c) and 2(d) for the Assisting and the Opposing configurations, respectively. Both figures point out a poor effect of the inclination angle on the wall temperature since the forced flow is anyway prevailing. In fact the belt velocity is at all angles larger than the transition velocity. Figures 2(c) and 2(d), for $\left|V_{b}\right|=1 \mathrm{~m} / \mathrm{s}$, exhibit also wall temperatures lower than those in Figs. 2(a) and 2(b), for $\left|V_{b}\right|=0.1 \mathrm{~m} / \mathrm{s}$, as a result of the larger velocity of the air, determined by the stronger flow dragged by the moving plate.

A less marked effect of the belt velocity is to be expected when the channel spacing is larger $(b=20 \mathrm{~mm})$, for which predictions are presented in Fig. 3 . Figure 3(a) shows, for the Assisting mode, lower heated wall temperatures than in the channel with $b=10 \mathrm{~mm}$, because of the larger air flow rate dragged in the channel through its larger inlet section. Figure 3(b) points out that, also in the larger channel, wall temperatures in the Opposing mode are larger than those in the Assisting one, since the buoyancy air flow is slowed up by the opposing belt velocity.

Figures 3(c) and 3(d) show that also for $b=20 \mathrm{~mm}$ the effect of the tilting angle almost vanishes passing from $\left|V_{b}\right|=0.1 \mathrm{~m} / \mathrm{s}$ to $\left|V_{b}\right|=1 \mathrm{~m} / \mathrm{s}$ in both configurations. The comparison of Figs. 3(c) and 3(d) to Figs. 2(c) and 2(d) points out slightly larger wall temperatures in the larger channel as a result of the larger distance of the belt from the heated wall.

Predictions for the case with a larger wall heat flux, $q_{w}=120 \mathrm{~W} / \mathrm{m}^{2}$, all other quantities being the same, are presented in Fig. 4. Buoyancy forces and, as a consequence, also the tilting angle, are expected to play a major role than in the afore presented cases. Figure 4(a) shows a fair dependence of heated wall temperatures on the inclination angle in the $\varphi=0^{\circ}-30^{\circ}$ range, a nearly $5 \mathrm{~K}$ maximum temperature increase passing to $\varphi=60^{\circ}$, whereas it is about $40 \mathrm{~K}$ in 

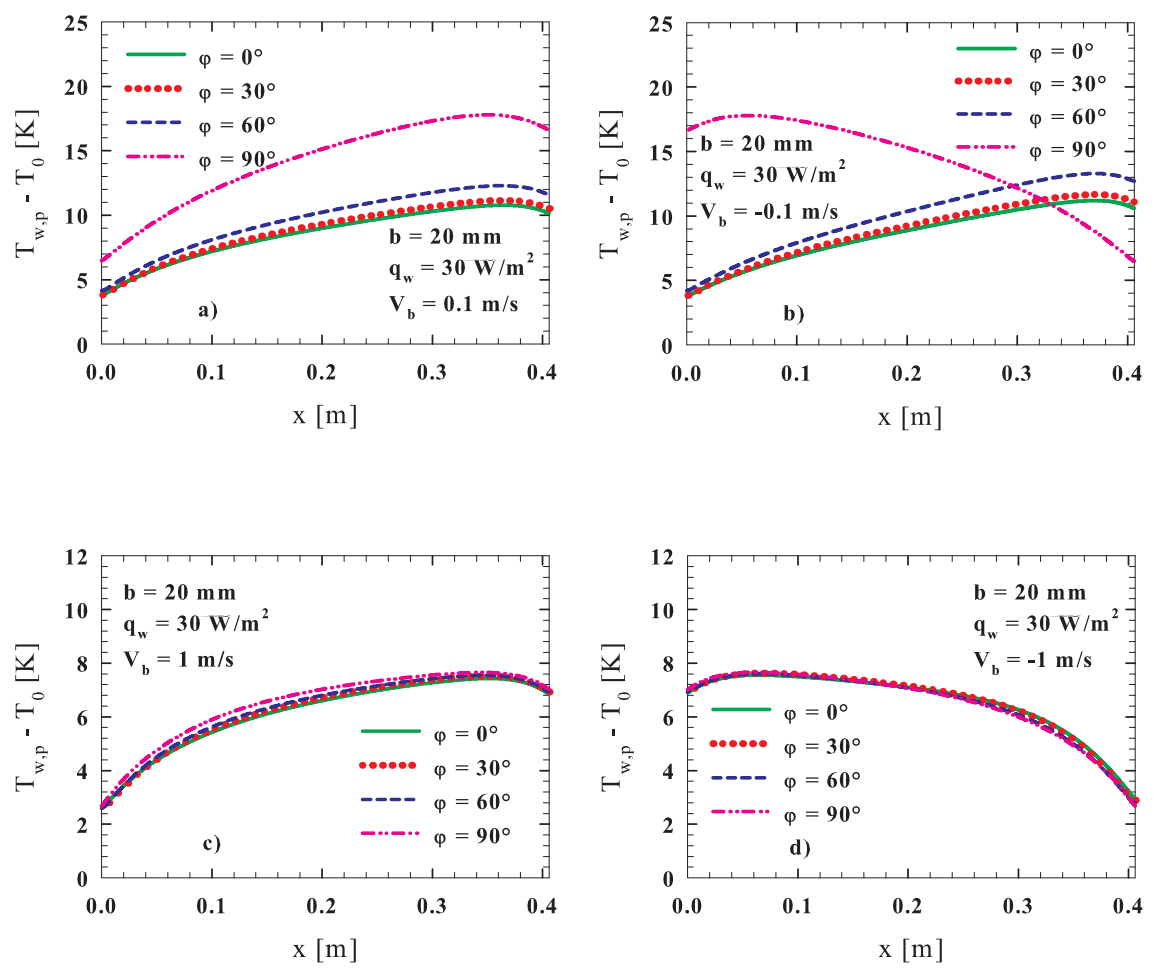

Figure 3: Heated wall temperature profiles vs axial coordinate for different inclination angles, $b=20 \mathrm{~mm}$ and $q_{w}=30 \mathrm{~W} / \mathrm{m}^{2}$ : (a) $V_{b}=0.1 \mathrm{~m} / \mathrm{s}$; (b) $V_{b}=-0.1 \mathrm{~m} / \mathrm{s}$; (c) $V_{b}=1 \mathrm{~m} / \mathrm{s}$; (d) $V_{b}=-1 \mathrm{~m} / \mathrm{s}$.

the horizontal channel. The comparison between Figs. 4(a) and 4(b) for inclined channels points out practically coincident wall temperature profiles that turn out to be independent of the direction of the belt velocity, since the contribution of the buoyancy forces is far larger than that of the forced flow when the unheated plate moves at low velocity $\left(\left|V_{b}\right|=0.1 \mathrm{~m} / \mathrm{s}\right)$, that is lower than the transition velocity.

Figures 4(c) and 4(d) exhibit a stronger dependence of wall temperature on the angle than in previous cases with $\left|V_{b}\right|=1 \mathrm{~m} / \mathrm{s}$, thus indicating that the larger wall heat flux allows buoyancy forces to play a significant role. Figure 4(c) points out that the larger the angle the larger the wall temperature, though the effect is less marked than in cases with $\left|V_{b}\right|=0.1 \mathrm{~m} / \mathrm{s}$. Figure 4(d) shows that in the Opposing configuration air flows upward in the channel for $\varphi=0^{\circ}$ and $\varphi=30^{\circ}$ and this indicates that in these cases the transition velocity is larger than the belt velocity. On the contrary, air flows downward for $\varphi=60^{\circ}$ and this allows to conclude that a $-1 \mathrm{~m} / \mathrm{s}$ transition velocity occurs for an inclination angle between $30^{\circ}$ and $60^{\circ}$. It is worth noticing that, once the transition occurred, the larger the angle the lower 

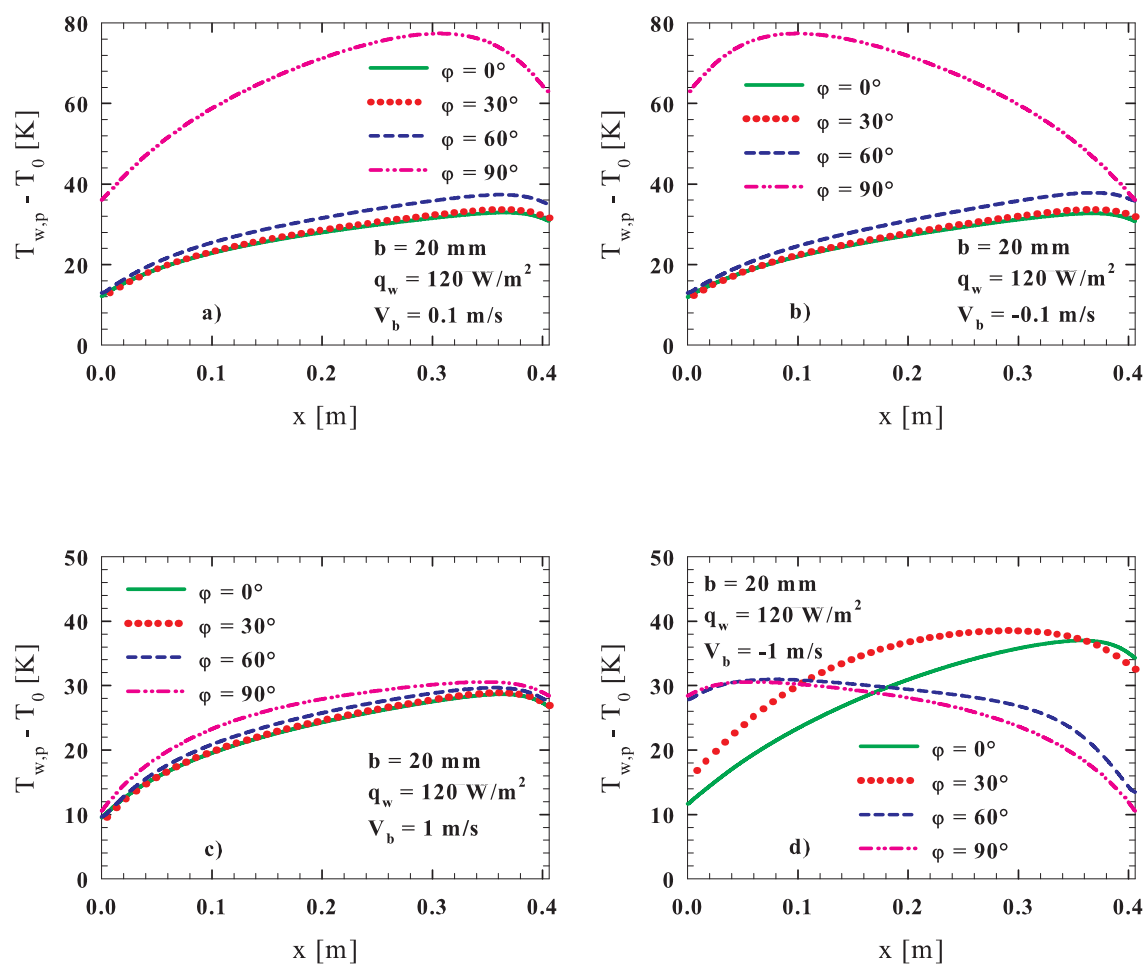

Figure 4: Heated wall temperature profiles vs axial coordinate for different inclination angles, $b=20 \mathrm{~mm}$ and $q_{w}=120 \mathrm{~W} / \mathrm{m}^{2}$ : (a) $V_{b}=$ $0.1 \mathrm{~m} / \mathrm{s}$; (b) $V_{b}=-0.1 \mathrm{~m} / \mathrm{s}$; (c) $V_{b}=1 \mathrm{~m} / \mathrm{s}$; (d) $V_{b}=-1 \mathrm{~m} / \mathrm{s}$.

the heated wall temperature. In fact, when forced flow overcomes the buoyancy forces and the air flow is descending, an increase in the inclination angle decreases the latter forces and increases the fluid velocity, thus lowering wall temperatures. We can conclude that, all other parameters being the same, the inclination angle at which the belt velocity equals the transition velocity decreases at increasing wall heat fluxes and at decreasing channel gaps.

Moving plate temperature profiles, referred to an ambient temperature equal to $300 K$, for the same values of the investigated quantities and directions of the belt velocity as those in Figs. 2-4, are presented in Figs. 5 and 6. Comparing Figs. 24 and 5-6 for the same conditions one can notice that, as it was to be expected, belt temperatures are in all cases lower than heated wall temperatures. It is worth remembering that the effect of radiative heat transfer is neglected and, therefore, the temperature of the unheated plate is affected only by the temperature of the flowing air that, in its turn, depends on the temperature of the heated wall.

Figure 5(a) shows that, in the Assisting configuration, in an inclined channel the larger the angle the warmer the unheated moving plate, like it occurs on the heated 

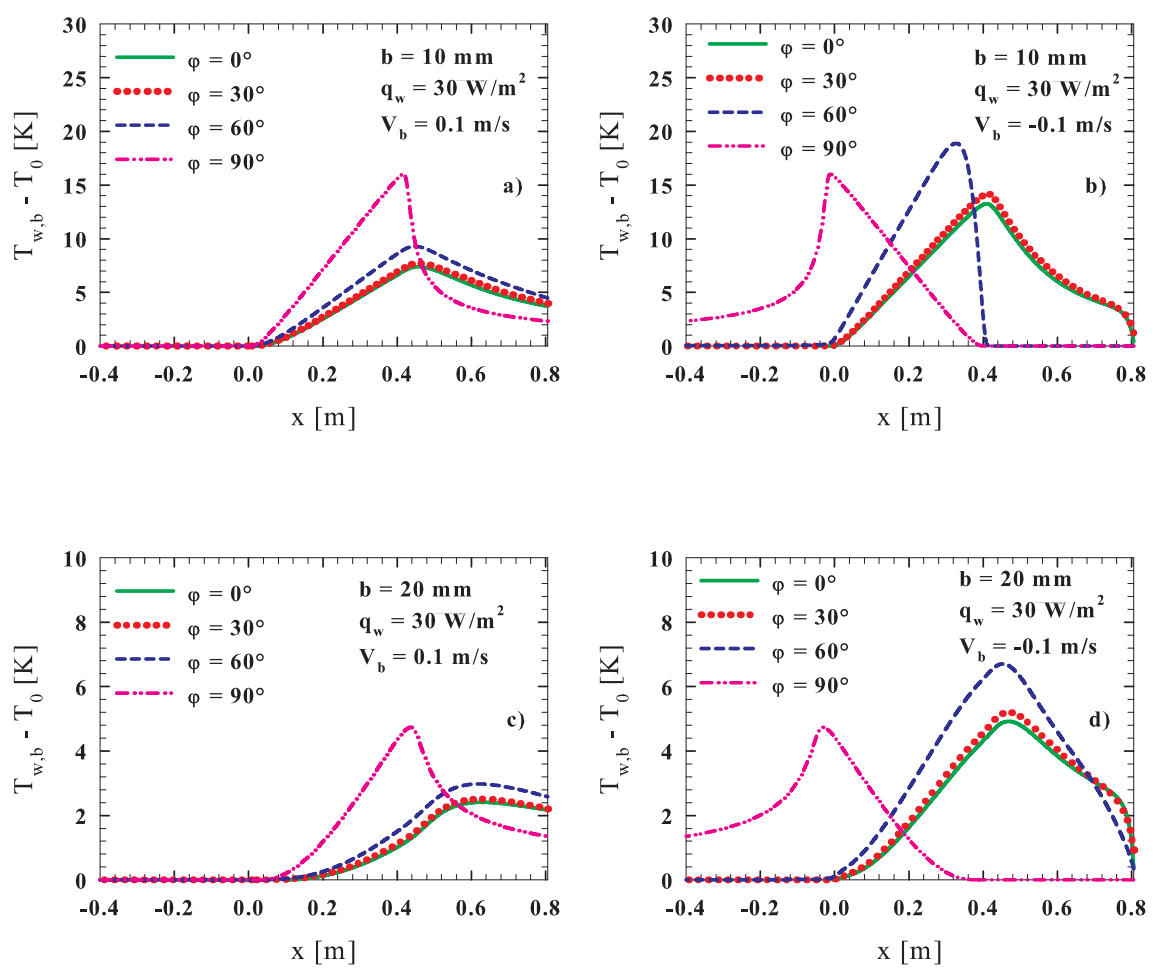

Figure 5: Belt temperature profiles vs axial coordinate for different inclination angles, $\left|V_{b}\right|=0.1 \mathrm{~m} / \mathrm{s}$ and $q_{w}=30 \mathrm{~W} / \mathrm{m}^{2}$ : (a)-(b) $b=10 \mathrm{~mm}$; (c) - (d) $b=20 \mathrm{~mm}$.

wall. We can remark that the temperature rise of the belt starts in the proximity of the entrance section of the channel $(x=0)$ and that the starting point shifts toward $x=0$ at increasing $\varphi$ values. One can also notice that the belt temperature increase continues downstream of the channel, since warm air still flows past the belt also downstream of its exit section. On the contrary, in a horizontal channel the belt cooling starts just in the exit section since the hot air leaving the channel moves upward and, consequently, the cooler ambient air flows past the belt immediately downward of the exit section. As far as the Opposing mode is concerned, Fig. 5(b) shows that for $\varphi=0^{\circ}$ and $\varphi=30^{\circ}$ air flow close to the belt is ascending since the larger $x$ the larger the belt temperature. In this case the increase in the belt temperature begins at the inlet section of the channel. The temperature profile in a $60^{\circ}$ inclined channel is nearly symmetric with reference to the mid-height section of the channel and this indicates a descending air flow and a belt velocity close to the transition velocity. Maximum belt temperature is attained for $\varphi=60^{\circ}$, just like it occurs at the heated wall temperature. 

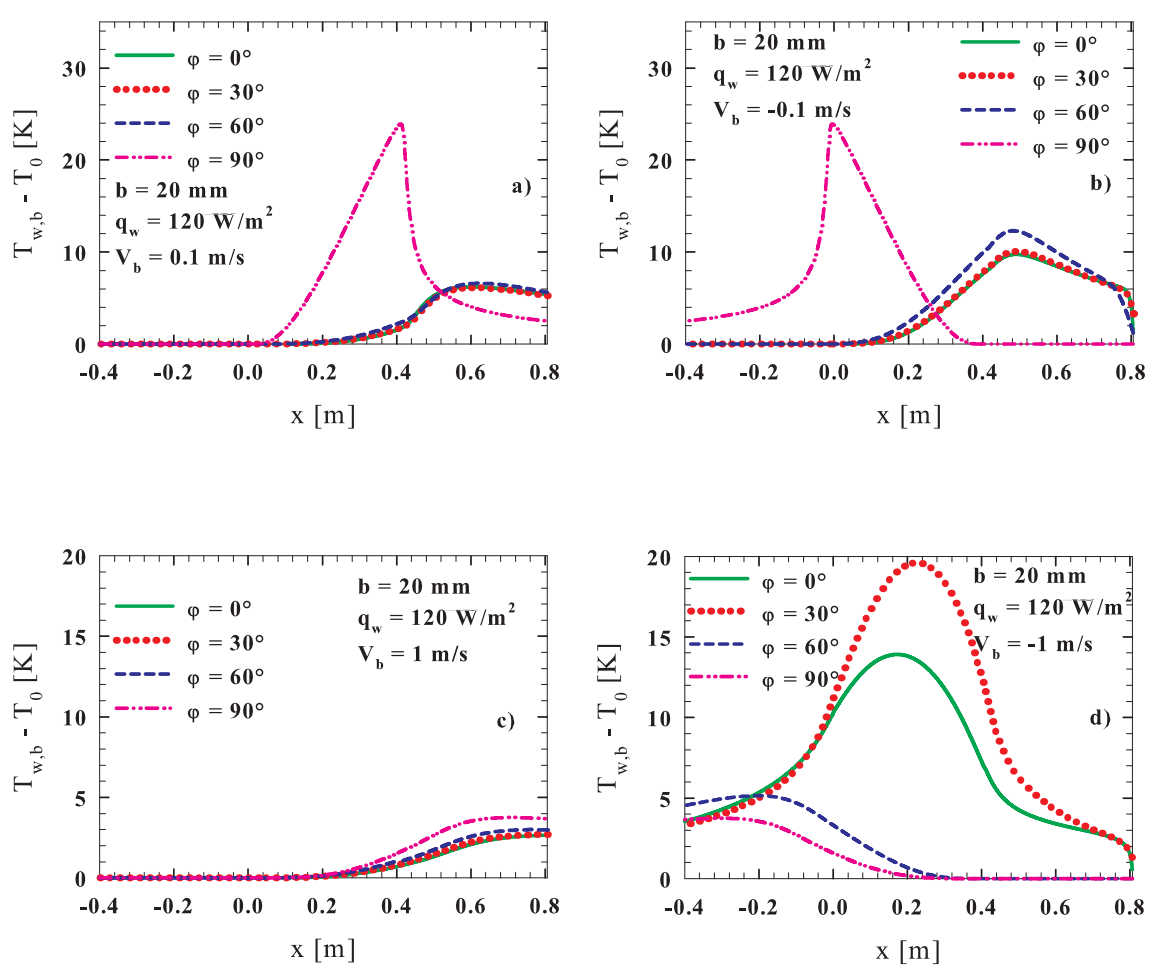

Figure 6: Belt temperature profiles vs axial coordinate for different inclination angles, $b=20 \mathrm{~mm}$ and $q_{w}=120 \mathrm{~W} / \mathrm{m}^{2}$ : (a) $V_{b}=0.1 \mathrm{~m} / \mathrm{s}$; (b) $V_{b}=-0.1 \mathrm{~m} / \mathrm{s}$; (c) $V_{b}=1 \mathrm{~m} / \mathrm{s}$; (d) $V_{b}=-1 \mathrm{~m} / \mathrm{s}$.

The comparison of Figs. 5(a) and 5(b) to Figs. 5(c) and 5(d) shows that increasing the channel spacing strongly decreases belt temperatures. Still comparing Figs. 5(a) and 5(c), we can notice that in the Assisting mode in inclined channels increasing the channel spacing increases both the $x$ coordinate where the heating of the moving plate begins and that where the maximum temperature of the belt is attained. The shift of the section where in the larger channel the belt temperature starts to increase is likely due to the larger distance between the inlet section of the channel and the section where the boundary layer fills it.

Figure 5(d) points out that, in the Opposing mode, in inclined channels air flows upward in the main. Observing Figs. 5(b) and 5(d) one can remark that in the $60^{\circ}$ inclined channel the difference between the belt velocity and the transition velocity for $b=20 \mathrm{~mm}$ is larger than that for $b=10 \mathrm{~mm}$, since the effect of drag forces is lower in the larger channel.

Unheated moving plate temperature profiles, referred to an ambient air temperature of $300 \mathrm{~K}$, for $b=20 \mathrm{~mm}, q_{w}=120 \mathrm{~W} / \mathrm{m}^{2},\left|V_{b}\right|=0.1 \mathrm{~m} / \mathrm{s}$ 
and $\left|V_{b}\right|=1 \mathrm{~m} / \mathrm{s}, \varphi=0^{\circ}, 30^{\circ}, 60^{\circ}, 90^{\circ}$, are presented in Fig. 6. Figure 6(a) exhibits a marked increase in the belt temperature downstream of the exit section of an inclined channel. This indicates that the temperature of the air leaving the channel is still warmer enough than that of the ambient air. Like already noticed previously, this does not occur in the horizontal channel.

Figures 6(c) and 6(d), compared to Figs. 6(a) and 6(b), show a stronger dependence of belt temperature profiles on the inclination angle in the Opposing than in the Assisting configuration. Let us notice that for $V_{b}=-1 \mathrm{~m} / \mathrm{s}$ the maximum belt temperature is attained for $\varphi=30^{\circ}$ and that in this case the belt temperature profile is nearly symmetric with reference to the mid-height section of the channel. This means that the velocity of the air close to the moving plate is very low and the transition nearly occurs. In fact far lower belt temperatures exhibited at $\varphi=60^{\circ}$ indicate that transition is already occurred.

\section{Conclusions}

A numerical investigation of mixed convection in air due to the interaction between the buoyancy flow and the forced flow induced by a moving plate in an inclined channel was carried out. The moving plate had a constant velocity and was unheated, whereas the other plate was stationary and heated at a uniform heat flux. The numerical solution was obtained by means of the commercial Fluent CFD code. Two configurations were analyzed: Assisting, where the vertical component of the belt velocity is opposed to the acceleration due to the gravity, and Opposing, where the afore mentioned component has the same direction as that of the acceleration due to the gravity. In the Opposing configuration the air in the channel flowed upward when the buoyancy forces prevailed on the drag forces whereas it moved downward when the drag forces prevailed on the buoyancy ones. The velocity at which the effects of the two kind of forces are quite the same was called transition velocity. Results showed that for the Assisting configuration the larger the inclination angle the larger the heated wall temperature, since the larger the inclination angle the smaller the contribution of the buoyancy force to the ascending air flow. This effect was more marked for $30^{\circ} \leq \varphi \leq 60^{\circ}$ than for $0^{\circ} \leq \varphi \leq 30^{\circ}$.

As far as the Opposing configuration is concerned, the air flow in the channel was ascending for $V_{b}=-0.1 \mathrm{~m} / \mathrm{s}$ and $0^{\circ} \leq \varphi \leq 60^{\circ}$, thus indicating that for these configurations the transition velocity was larger than the belt velocity. The analysis of results for $V_{b}=-1 \mathrm{~m} / \mathrm{s}$ shows that the inclination angle at which the belt velocity became equal to the transition one decreased at increasing wall heat fluxes and at decreasing channel gaps.

\section{Nomenclature}

$b$

$g$

$k$ channel spacing, $m$

acceleration due to the gravity, $\mathrm{m} / \mathrm{s}^{2}$

kinetic energy of turbulence, $\mathrm{m}^{2} / \mathrm{s}^{2}$ 


$\begin{array}{ll}L & \text { length, } m \\ q_{w} & \text { wall heat flux, } W / m^{2} \\ s & \text { heated plate thickness, } m \\ T & \text { temperature, } K \\ V_{b} & \text { belt velocity, } m / s \\ x, y & \text { Cartesian coordinates, } m\end{array}$

Greek symbols

$\begin{array}{ll}\varepsilon & \text { dissipation rate of turbulent kinetic energy, } \mathrm{m}^{2} / \mathrm{s}^{3} \\ \varphi & \text { inclination angle, degree }\end{array}$

Subscripts

$\begin{array}{ll}0 & \text { ambient } \\ b & \text { belt (moving plate) } \\ p & \text { plate } \\ w & \text { wall } \\ x, y & \text { along the Cartesian coordinates }\end{array}$

\section{Acknowledgement}

This work was supported by MIUR with a 2005 PRIN grant.

\section{References}

[1] Jaluria, Y., Transport from continuously moving materials undergoing thermal processing (Chapter 4). Annual Review of Heat Transfer, ed. C.L. Tien, Hemisphere Publ. Co.: New York, pp. 187-245, 1992.

[2] Viskanta, R. \& Bergman, T. L., Heat transfer in materials processing. Handbook of Heat Transfer, McGraw-Hill: New York, 3rd edition, 1998.

[3] Jaluria, Y., Fluid flow phenomena in material processing. Journal of Fluid Engineering, 123, pp. 173-210, 2001.

[4] Jaluria, Y., Thermal processing of materials: from basic research to engineering. Journal of Heat Transfer, 125 (6), pp. 957-979, 2003.

[5] Jaluria, Y. \& Kang, B. H., Heat transfer from continuously moving material in channel flow for thermal processing. Journal of Thermophysics and Heat Transfer, 8 (3), pp. 546-554, 1994.

[6] Andreozzi, A., Bianco, N., Manca, O. \& Naso, V., Mixed Convection Heat Transfer in a Vertical Channel with a Moving Plate. Proceedings of 12th International Conference of Computational Methods and Experimental Measurements, pp.695-704, 2005.

[7] Andreozzi, A., Bianco, N., Manca, O. \& Naso, V., Numerical Analysis of Opposing Mixed Convection in Air in a Vertical Channel with a Moving Plate. Proceedings of International Mechanical Engineering Congress and Exposition (ASME-IMECE), paper n.IMECE2005-82983, 2005. 
[8] Andreozzi, A., Bianco, N., Lacasa, G. \& Naso, V., Experimental Analysis of Mixed Convection in Vertical Channels with a Moving Plate. Proceedings of Energy: Production, Distribution and Conservation ASME ATI Conference, II pp. 833-842, 2006.

[9] Andreozzi, A., Manca, O. \& Naso, V., Thermal and Fluid Dynamic Behavior of a Horizontal Channel with Adiabatic Moving Lower Plate. Proceedings of International Mechanical Engineering Congress and Exposition (ASMEIMECE), paper n.IMECE2005-82259, 2005.

[10] Fluent Incorporated, Fluent 6.1, User Manual, Lebanon, NH, 2003. 